\title{
What is the order of the deconfining phase transition?
}

\author{
A. Di Giacomo ${ }^{\mathrm{a}}$ \\ ${ }^{\text {a}}$ Dipartimento di Fisica, Università di Pisa, \\ I.N.F.N. Sezione di Pisa
}

The techniques are discussed by which the order of the deconfining phase transition is investigated on the lattice. QCD with $N_{f}=2$ is a special case, which can provide information on the mechanism of confinement.

\section{Introduction}

Quarks and gluons have never been observed as free particles, a phenomenon known as color confinement.

A widely accepted idea in the 1960's was that a limiting temperature exists for hadronic matter [1], the Hagedorn temperature $T_{H}$ : increasing the energy density just below $T_{H}$ would result in production of particles, rather than in an increase of temperature. In ref. 2] it was suggested that a phase transition could exist to a plasma of quarks and gluons (Deconfining Transition).

Big experiments to explore this possibility in heavy ions collisions have not yet given conclusive results 3 .

The only existing source of information are Montecarlo simulations of the theory on the Lattice.

The partition function of a system of fields at temperature $\mathrm{T}$

$Z=\operatorname{Tr}\{\exp (-H / T)\}$

is equal to the Euclidean Feynmann integral of the theory extending in time from 0 to $1 / T$, with periodic boundary conditions in time for bosons, antiperiodic for fermions. In the Lattice formulation this corresponds to simulate the theory on a lattice $N_{s}^{3} \times N_{t}$, with the spatial extension $N_{s} \gg N_{t}$, the time extension. The temperature is $T=1 / a\left(\beta, m_{q}\right) N_{t}$, where $\beta=2 N / g^{2}$ and $a$ is the lattice spacing in physical units.

To explore the deconfining transition a signature for confinement-deconfinement must be de- fined. In quenched QCD $\left(N_{f}=0\right.$, no dynamical quarks) this is done as follows. Consider the Polyakov line

$L(\vec{x})=P \exp \left(i \int_{0}^{1 / T} A_{0}(\vec{x}, t) d t\right)$

The correlator

$G(\vec{r})=\left\langle L(\vec{r}) L^{\dagger}(0)\right\rangle$

is related to the static $q \bar{q}$ potential by the relation

$V(\vec{r})=-T \ln G(\vec{r})$

On the other hand at large distances cluster property requires

$G(\vec{r}) \underset{r \rightarrow \infty}{\simeq} c \exp \left(-\frac{\sigma}{T} r\right)+|\langle L\rangle|^{2}$

When $\langle L\rangle=0$ eq(5) gives a linearily rising potential at large distances, $V=\sigma r$ which is taken as a definition of confinement. When $\langle L\rangle \neq 0$, $V(r) \rightarrow$ const and there is no confinement. $\langle L\rangle$ is an order parameter for confinement, the corresponding symmetry being $Z_{3}$.

A deconfining phase transition is indeed observed in lattice simulations, corresponding to the above definition, both in $S U(2)$ and in $S U(3)$ pure gauge theories: $\langle L\rangle$ grows rapidly from 0 to 1 by increasing $T$ at some $T_{c}$. In a finite lattice, however, the growth of $\langle L\rangle$ is smooth: no phase transition can take place in a finite system [4]. The raise of $\langle L\rangle$ becomes sharper and sharper as the volume increases.

The infinite volume limit can be studied by a technique used in statistical mechanics [5], known 
as finite size scaling analysis. The susceptibility of the order parameter $\chi_{L}$ can be defined at a given spatial size $N_{s}$

$\chi_{L}\left(N_{s}\right)=\int d^{3} x\left\langle L(x) L^{\dagger}(0)-L(0) L^{\dagger}(0)\right\rangle$

$\chi_{L}$ gives a measure of the slope of the increase of $\langle L(x)\rangle$ at $T_{c}$. As $N_{s} \rightarrow \infty$ it diverges with some critical index $\gamma$ when approaching $T_{c}$ from below

$\chi_{L}\left(N_{s}\right) \underset{T \rightarrow T_{c}^{-}}{\simeq} \tau^{-\gamma} \quad \tau=\left(1-\frac{T}{T_{c}}\right)$

The correlation length $\xi$ of the order parameter $\langle L(x)\rangle$ also diverges at the critical point with a critical index $\nu$

$\xi \underset{T \rightarrow T_{c}^{-}}{\simeq} \tau^{-\nu}$

Assuming that the ratio

$R\left(N_{s}\right) \equiv \frac{\chi_{L}\left(N_{s}\right)}{\chi_{L}(\infty)}=f\left(\frac{a}{\xi}, \frac{a N_{s}}{\xi}\right)$

is an analytic function, as $T \rightarrow T_{c}, a / \xi \rightarrow 0$ and the scaling law follows

$R\left(N_{s}\right) \simeq f\left(0, \frac{a N_{s}}{\xi}\right)$

or

$\chi_{L}\left(N_{s}\right) \simeq N_{s}^{\gamma / \nu} \Phi_{L}\left(N_{s}^{1 / \nu} \tau\right)$

The dependence on $N_{s}$ is dictated by the critical indices of the transition. In particular the value of $\chi_{L}$ at the peak $\tau=0$ scales as

$\chi_{L, \text { peak }}\left(N_{s}\right) \propto N_{s}^{\gamma / \nu}$

A similar discussion gives for the specific heat $C_{v}$

$C_{v}=C_{v}^{0}+N_{s}^{\alpha / \nu} \Phi_{C}\left(N_{s}^{1 / \nu} \tau\right)$

with $\alpha$ the corresponding critical index and $C_{v}^{0}$ an additive term due to the presence of an additive renormalization. By studying numerically the dependence of $\chi_{L}$ and $C_{v}$ on $N_{s}$ the critical indices $\alpha, \gamma, \nu$ can be determined and the order of the transition and the universality class with them. A weak first order transition is a limiting case when $\alpha=\gamma=1$ and $\nu=1 / d$ with $d$ the number of spatial dimensions.
For quenched $S U(2)$ it is found that the universality class is that of the $3 d$ ising model, and $\nu=.62[6]$. For quenched $S U(3)$ the transition is weak first order [7] and $\nu=1 / 3$.

An alternative order parameter $\langle\mu\rangle$ is the vev of a magnetically charged operator $[8$. This operator will be described in some detail in the next section for full QCD. A finite size scaling analysis of

$\rho=\frac{d}{d \beta} \ln \langle\mu\rangle$

gives results consistent with those obtained by using $\langle L\rangle$ and $\langle\mu\rangle \neq 0$ for $T<T_{c},\langle\mu\rangle=0$ for $T>T_{c}$.

Since $\langle\mu\rangle \neq 0$ signals dual superconductivity, this result can be considered as evidence that dual superconductivity of the vacuum is the mechanism of confinement.

\section{Full QCD}

In the presence of dynamical quarks a clear way to define and to detect confinement does not exist. $\langle L\rangle$ is not a good order parameter, since the corresponding symmetry $Z_{3}$ is broken by the quark coupling. In addition string breaking is expected to occur: the static potential energy converts into dynamical $q-\bar{q}$ pairs at large distances so that the potential is not linear any more with the distance, even if there can be confinement.

At $m_{q}=0$ chiral symmetry exists, which is spontaneously broken at $T=0$, the pseudoscalar mesons being the corresponding Goldstone particles and the quark condensate $\langle\bar{\psi} \psi\rangle$ the order parameter. At some critical temperature $T_{c} \sim 170 \mathrm{Mev}$ chiral symmetry is restored. It is not well understood what is the interplay of chiral symmetry with confinement. Moreover at $m_{q} \neq 0$ chiral symmetry is explicitely broken and $\langle\bar{\psi} \psi\rangle$ is not a good order parameter either.

However if one looks at susceptibilities like $\chi_{L}, \chi_{\bar{\psi} \psi}, C_{v}$ they all show in numerical simulations a maximum for a $T_{c}\left(m_{q}\right)$ as functions of $T$, along a line in the plane $\left(m_{q}, T\right)$, which is assumed by convention as a critical line, with the confined phase below it and the deconfined phase above it 9 [10]. 


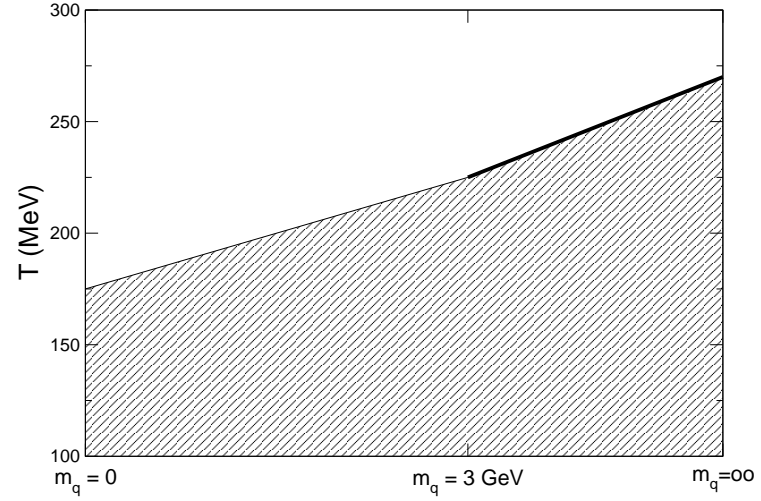

Figure 1. Phase diagram of two flavor QCD

We shall discuss a simplified model with $N_{f}=$ 2 and $m_{u}=m_{d}=m$, which is semirealistic, but is of special interest as a probe of the theory. The critical line is schetched in fig 1 . for this model.

A renormalization group analysis of the chiral transition at $m=0$ [1] leads to the following predictions, based on the hypothesis that the Golstone particles are the relevant degrees of freedom at the transition. For $N_{f}=3$ or $2+1$ the transition is first order, and such is expected to be for $m \neq 0$. For $N_{f}=2$ the transition is first order both at $m=0$ and at $m \neq 0$ if the axial $U(1)$ broken by the anomaly is restored at a lower temperature than chiral symmetry, it is second order if the anomaly persists up to the chiral transition, and in that case the line in fig. 1 is a crossover . There exists no precise numerical analysis of the order of the transition, but for some reason the second possibility is usually accepted.

One can assume dual superconductivity of the vacuum as a criterion for confinement. This criterion works in the quenched case, where it is consistent with the one based on $\langle L\rangle$ [8]. To be rigorous one should prove that dual superconductivity implies confinement of any colored particle . In the same way, however, one should prove that $\langle L\rangle=0$ implies confinement of any colored particle.

Dual superconductivity is detected by a disorder parameter $\left\langle\mu^{a}\right\rangle$, the vev of an operator carry- ing non zero magnetic charge 8 . A non zero vev of $\mu^{a}$ signals Higgs breaking of magnetic U(1) symmetry, or dual superconductivity. We shall recall below the definition and some properties of $\mu^{a}$. Numerical simulations show indeed that vacuum is a dual superconductor in the region of fig 1 below the critical line, and is normal above it 8

The operator $\mu^{a}$ is defined 12 by the orbits $U(x) \phi_{\text {diag }}^{a} U^{\dagger}(x)$ in the gauge group of the symmetric space defined by the co-roots

$\phi_{\text {diag }}^{a}=\operatorname{diag}(\overbrace{\frac{N-a}{N}, . ., \frac{N-a}{N}}^{a}, \overbrace{-\frac{a}{N}, . .,-\frac{a}{N}}^{N-a})$

The definition is

$\mu^{a}(\vec{x}, t)=e^{i \int d^{3} \vec{y} \operatorname{Tr}\left(\phi^{a}(\vec{y}, t) \vec{E}(\vec{y}, t)\right) \vec{b}_{\perp}(\vec{x}-\vec{y})}$

Each choice of $U(x)$ defines an abelian projection [13], and with it monopoles. The operator $\mu^{a}$ creates a monopole in the given abelian projection. It can be shown 14 15], however, that the property of $\left\langle\mu^{a}\right\rangle$ of being non-zero or zero is abelian projection independent,if the number density of monopoles is finite. Numerical simulations show that this is indeed the case. If one looks at the distribution of the difference of eigenvalues of operators in the adjoint representation on the lattice sites, the number of sites in which that difference vanishes is zero. That difference is equal to zero at the locations of monopoles. A large sample of configurations, lattice spacings, and operators have been studied.

A finite size scaling analysis allows to determine the critical indices and the order of the transition 16] The problem has two scales, the correlation length and the quark mass so that neglecting the ratio of the lattice spacing to the correlation length,

$\langle\mu\rangle=f\left(\frac{N_{s}}{\xi}, m N_{s}^{y_{h}}\right)$

where $y_{h}$ is the anomalous dimension of $m$. By choosing values of $m$ and $N_{s}$ such that the second argument is constant a scaling law follows for the susceptibility

$\rho=\frac{d}{d \beta} \ln \langle\mu\rangle \quad \rho=N_{s}^{1 / \nu} \Phi\left(N_{s} \tau^{1 / \nu}\right)$ 
whence $\nu$ can be extracted. The result is consistent with a first order thansition $(\nu=1 / 3)$ [17.

This determination should be consistent with the scaling of the specific heat eq(12). Preliminary data show that this is the case indeed 17. Further numerical work is on the way to put the result on a firm basis. This would definitely legitimate the assumption of $\langle\mu\rangle$ as an order parameter for confinement, and confirm dual superconductivity as a mechanism for color confinement. It would also imply[11] that either axial $U(1)$ symmetry is restored before chiral symmetry, or that Goldstone particles are not the relevant degrees of freedom at the critical point. The first possibility can also be tested on the lattice.

This work is partially supported by MIUR Progetto Teoria delle interazioni fondamentali. Thanks are due to my collaborators J.M. Carmona, L.Del Debbio, M. D'Elia, B. Lucini, G. Paffuti, C. Pica for discussions .

\section{REFERENCES}

1. R. Hagedorn Nuovo Cim.Suppl. 3 (1965) 147

2. N.Cabibbo, G. Parisi Phys.Lett.B59:67,1975

3. See e. g. Proceedings Quark Matter 2002 H.Gutbrot,J.Aichelin and K.Werner ed.s North Holland

4. C.N.Yang, T.D. Lee Phys.Rev.87:404409,1952

5. M.E. Fischer, M.N. Barber Phys.Rev. Lett. 2815161973

6. J. Fingberg, U. M. Heller, F. Karsch, Nucl. Phys. B392, 493 (1993).

7. M. Fukugita, M. Okawa, A. Ukawa, Phys. Rev. Lett. 631768 (1989).

8. L. Del Debbio, A. Di Giacomo, G. Paffuti, Phys. Lett. B 349 , 513 (1995); A. Di Giacomo, B. Lucini, L. Montesi, G. Paf- futi, Phys. Rev. D 61 , 034503 (2000); A. Di Giacomo, B. Lucini, L. Montesi, G. Paf- futi, Phys. Rev. D 61 , 034504 (2000);J.M. Carmona, M. D'Elia, A. Di Giacomo, B. Lucini, G. Paffuti, D 64, 114507 (2002).

9. F. Karsch and E. Laermann, Phys. Rev. D 50 , 6954 (1994)

10. S. Aoki et al. (JLQCD collaboration), Phys. Rev. D 57,3910 (1998).
11. R. Pisarski and R. Wilczek, Phys. Rev. D 29 , 338 (1984).

12. L.Del Debbio, A.Di Giacomo, B.Lucini, G.Paffuti Abelian projection for $\mathrm{SU}(\mathrm{N})$ gauge theory heo-lat/0203023

13. G.'tHooft Nucl.Phys.B190 4551981

14. A. Di Giacomo,Independence of dual superconductivity on the abelian projection hep-lat/0206018

15. A. Di Giacomo, G. Paffuti The abelian projection revisited hep-lat/0309019 (LATTICE 2003)

16. J.M. Carmona, M. D'Elia, L. Del Debbio, A. Di Giacomo, B. Lucini, G. Paffuti, Phys. Rev. D 66,011503 (2002).

17. J.M.Carmona, M. D'Elia, L.Del Debbio, A. Di Giacomo, B. Lucini, G.Paffuti, C.Pica (LATTICE 2003) hep-lat/ 0309035 